\title{
Fuzzy Domain Decomposition: a new perspective on heterogeneous DD methods
}

\author{
Martin J. Gander ${ }^{1}$ and Jérôme Michaud ${ }^{1}$
}

\section{Motivation}

In a wide variety of physical problems, the complexity of the physics involved is such that it is necessary to develop approximations, because the complete physical model is simply too costly. Sometimes however the complete model is essential to capture all the physics, and often this is only in part of the domain of interest. One can then use heterogeneous domain decomposition techniques: if we know a priori where an approximation is valid, we can divide the computational domain into subdomains in which a particular approximation is valid and the topic of heterogeneous domain decomposition methods is to find the corresponding coupling conditions to insure that the overall coupled solution is a good approximation of the solution of the complete physical model. For an overview of such techniques, see $[9,10]$ and references therein. However, there are many physical problems where it is not a priori known where which approximation is valid. In such problems, one needs to track the domain of validity of a particular approximation, and this is usually not an easy task. An example of such a method is the $\chi$-method, see $[4,1]$.

In this contribution, we introduce a new formalism for heterogeneous domain decomposition, which is not based on a sharp decomposition into subdomains where different models are valid. The main idea relies on the notion of Fuzzy Sets introduced by Zadeh [12] in 1965. The Fuzzy Set Theory relaxes the notion of belonging to a set through membership functions to (fuzzy) sets that account for partially belonging to a set. In the context of heterogeneous domain decomposition, this could be useful if one assumes that the computational domain can be decomposed into fuzzy sets that form a partition of the domain in a sense that needs to be specified. Once such a partition is given, one can compute the solution of the coupled problem using the membership functions. Note that the membership functions can depend on space and time and therefore can take into account a change in the validity domain of a particular approximation. We show here that this technique leads to an excellent coupling strategy for the 1D advection dominated diffusion problem. Such a domain decomposition method would be able, in principle, to take into account part of the domain where none of the available approximations are valid under the assumption that a combination of them is a good enough approximation there.

On the assumption $\mathbf{u}=\mathbf{u}_{1}+\mathbf{u}_{2}$ : The idea to use fuzzy set theory came from an assumption that arose in some specific coupling methods (see below). We formulate it here for a generic partial differential equation of the form

Université de Genève, 2-4 rue du Lièvre, CP 64, CH-1211 Genève 4,

\{Martin.Gander, Jerome.Michaud\}@unige.ch 


$$
\mathscr{L}(u)=g,
$$

where $\mathscr{L}$ is a linear differential operator.

Assumption $1\left(u=u_{1}+u_{2}\right)$ We assume that the solution $u$ of $(1)$ can be written as a sum, $u=u_{1}+u_{2}$, and that one can derive a coupled system for the new unknowns $u_{1}$ and $u_{2}$. The derivation of the coupled system might then use relevant approximations for one or both components.

This assumption has been used at least in two different series of papers: the first one is in physics for the approximation of neutrino radiative transfer in core-collapse supernovae $[11,2,3]$, and the second one is in mathematics for the coupling between the kinetic equation and approximations of it (diffusion, Euler, Navier-Stokes...) $[8,5,6,7]$.

In the following, we will see how this assumption can be linked with fuzzy sets. This will lead us to introduce fuzzy domain decomposition methods.

\section{Fuzzy Sets and Fuzzy Domain Decomposition Methods}

Let $X$ be a set in the classical sense of generic elements $x$, such that $X=\{x\}$.

Definition 1 (Fuzzy Set). A fuzzy set $A$ of $X$ is characterized by a membership function $h_{A}(x)$ that associates to every point of $X$ a real number in $[0,1]$. The value of $h_{A}(x)$ represents the grade of membership of $x$ in $A$. The support $\operatorname{Supp}(A)$ of a fuzzy set A is the classical subset of $X$ defined by $\operatorname{Supp}(A)=\left\{x \in X \mid h_{A}(x) \neq 0\right\}$.

Remark 1. If the membership function is a characteristic function, then we recover the classical notion of sets.

We next list a few useful properties of fuzzy sets:

Definition 2 (Complementary set). The complementary set $A^{c}$ of a fuzzy set $A$ is defined by its membership function $h_{A^{c}}=1-h_{A}$.

Definition 3 (Union of fuzzy sets). The union of two fuzzy sets $A$ and $B$ of membership function $h_{A}(x)$ and $h_{B}(x)$ is the fuzzy set $C$, denoted by $C=A \cup B$. It is characterized by its membership function $h_{C}(x)$ linked with those of $A$ and $B$ by $h_{C}(x)=\max \left(h_{A}(x), h_{B}(x)\right), \forall x \in X$.

Remark 2. The union of a fuzzy set with its complementary set is not equal to the initial set, unless the membership functions are characteristic functions: $A \cup A^{c} \varsubsetneqq X$.

Definition 4 (Algebraic sum of fuzzy sets). The algebraic sum of $A$ and $B$ is denoted by $A+B$ and is defined by the membership function $h_{A+B}=h_{A}+h_{B}$. This definition has a meaning only if $h_{A}(x)+h_{B}(x) \leq 1, \forall x \in X$.

Remark 3. Note that the algebraic sum has the property that $A+A^{c}=X$. 
Let $\Omega$ be the computational domain of the problem we want to solve. We use the algebraical sum of fuzzy sets to obtain a decomposition of the domain:

Definition 5 (Fuzzy Domain Decomposition (FDD)). A fuzzy domain decomposition is given by the fuzzy sets $\Omega_{i}, i=1, \ldots, n$ defined by their membership functions $h_{i}$ such that their algebraic sum equals the domain $\Omega: \Omega=\Omega_{1}+\ldots+\Omega_{n}$. In terms of membership functions, this condition reads $\sum_{i=1}^{n} h_{i}(x)=1, \forall x \in \Omega$.

Definition 6. Let $u$ be a function from $\Omega$ to $\mathbb{R}$. We define the restriction of $u$ to the fuzzy set $A$ of $\Omega$ by $u_{A}=h_{A} u$, where $h_{A}$ is the membership function of $A$.

Proposition 1. Let $u$ be a function from $\Omega$ to $\mathbb{R}$, let $\left\{\Omega_{i}\right\}_{i=1}^{n}$ be a fuzzy domain decomposition of $\Omega$ and let $u_{i}$ be the restriction of $u$ to $\Omega_{i}$. Then

$$
u=\sum_{i=1}^{n} u_{i} \quad \text { and } \quad u^{\prime}=\sum_{i=1}^{n} u_{i}^{\prime} .
$$

Proof. This is a direct consequence of Definition 6 of the restriction of $u$ to fuzzy sets, and the linearity of derivatives.

Definition 7 (FDDM, eFDDM, iFDDM). A FDD method (FDDM), is a numerical method based on an FDD of the domain. We will say that an FDDM is explicit (eFDDM) if the membership functions $h_{i}$ are explicitly known, and implicit otherwise (iFDDM).

Remark 4. The relation (2) shows that if the Assumption 1 is used, it is natural to interpret the resulting method as an FDDM. The methods of Degond et al. [8, 5, 6, 7] belong to the eFDDM class, but the IDSA $[11,2,3]$ is an example of an iFDDM.

If we want to obtain an heterogeneous DDM, we need two ingredients. The first one is a coupling methodology between the two approximations (one of them may be exact), and the second one is a criterion to decide where an approximation is valid. The advantage of an eFDDM is that the $h_{i}$ functions are used both for implementing the coupling and the criterion. As the partition is explicitly known, we can change it to test various criteria for the validity of the different approximations.

We now show the coupling procedure for a decomposition into two fuzzy domains. Assume that we want to solve an approximation of Problem (1) and that we have two approximations $\mathscr{L}_{1}$ and $\mathscr{L}_{2}$ of the linear operator $\mathscr{L}$ valid in a fuzzy sense in $\Omega_{1}$ and $\Omega_{2}$ respectively. Then, we can decompose Problem (1) as

$$
\mathscr{L}\left(u^{*}\right)=g \quad \Leftrightarrow \quad h_{1} \mathscr{L}\left(u^{*}\right)+h_{2} \mathscr{L}\left(u^{*}\right)=g \quad \rightsquigarrow \quad h_{1} \mathscr{L}_{1}(u)+h_{2} \mathscr{L}_{2}(u)=g,
$$

where we have introduced in the last formulation the approximated operators. Here, $u^{*}$ stands for the exact solution and $u$ for the approximate solution. The symbol $\rightsquigarrow$ means "is approximated by". In order to obtain a FDDM, we will use Assumption 1, and to obtain an explicit method in the sense of Definition 7 , we require 


$$
u_{i}=h_{i} u, \quad u_{i}^{\prime}=h_{i}^{\prime} u+h_{i} u^{\prime}, \quad u_{i}^{\prime \prime}=h_{i}^{\prime \prime} u+2 h_{i}^{\prime} u^{\prime}+h_{i} u^{\prime \prime}, \quad i=1,2,
$$

where we used the product rule for $h_{i}$ sufficiently smooth.

As $g=h_{1} g+h_{2} g$, we can rewrite Equation (3) 3 as a system

$$
\left\{\begin{array} { l } 
{ h _ { 1 } \mathscr { L } _ { 1 } ( u ) = h _ { 1 } g \text { on } \Omega , } \\
{ h _ { 2 } \mathscr { L } _ { 2 } ( u ) = h _ { 2 } g \text { on } \Omega , }
\end{array} \rightsquigarrow \left\{\begin{array}{l}
\widetilde{\mathscr{L}}_{1}\left(u_{1}\right)=h_{1} g+\mathscr{L}_{12}\left(u_{2}\right) \text { on } \operatorname{Supp}\left(\Omega_{1}\right), \\
\widetilde{\mathscr{L}}_{2}\left(u_{2}\right)=h_{2} g+\mathscr{L}_{21}\left(u_{1}\right) \text { on } \operatorname{Supp}\left(\Omega_{2}\right) .
\end{array}\right.\right.
$$

The second system is obtained by using Assumption 1 and Equation (4). The use of the product rule to handle the fact that the $h_{i}$ do not commute with $\mathscr{L}_{i}$ leads to the operators $\widetilde{\mathscr{L}_{i}}$ and $\mathscr{L}_{i, 3-i}$ that are linked by the relation

$$
\widetilde{\mathscr{L}_{i}}=\mathscr{L}_{i}-\mathscr{L}_{i, 3-i}, \quad i=1,2
$$

The change in support simply reflects the fact that Equation $(5)_{1}$ is non-trivial only in $\operatorname{Supp}\left(\Omega_{i}\right)$. Equation (5) 2 is an eFDDM for Problem (3) 3 .

Remark 5 . The boundary conditions of an eFDDM can be easily defined by transferring the boundary conditions on $u$ to $u_{i}$ using Equation (4).

\section{An Example: Advection Dominated Diffusion}

As an example, we consider for $v, a>0$ the $1 \mathrm{D}$ advection diffusion equation

$$
\mathscr{L}\left(u^{*}\right)=v u^{* \prime \prime}+a u^{* \prime}=0 \quad \text { on }(0,1), \quad u^{*}(0)=0, \quad u^{*}(1)=1,
$$

whose closed form solution is given by $u^{*}(x)=\frac{e^{-a x / v}-1}{e^{-a / v}-1}$. For $\frac{v}{a} \ll 1$, the diffusion term is only important close to 0 where a boundary layer forms. We can define the operators

$$
\mathscr{L}_{1}:=\mathscr{L}=v \partial_{x x}+a \partial_{x}, \quad \text { and } \quad \mathscr{L}_{2}:=a \partial_{x},
$$

and, as before, using Assumption 1 and Equation (4) we have

$$
\mathscr{L}_{12}:=v\left(h_{1}^{\prime \prime}+2 h_{1}^{\prime} \partial_{x}\right)+a h_{1}^{\prime} \quad \text { and } \quad \mathscr{L}_{21}:=a h_{2}^{\prime} .
$$

The eFDDM method we get with the operators from $(8,9)$, using Equation (6) to define $\widetilde{\mathscr{L}_{i}}$, with $g=0$, is

$$
\begin{aligned}
v u_{1}^{\prime \prime}+\left(a-2 v h_{1}^{\prime}\right) u_{1}^{\prime}-\left(v h_{1}^{\prime \prime}+a h_{1}^{\prime}\right) u_{1} & =2 v h_{1}^{\prime} u_{2}^{\prime}+\left(v h_{1}^{\prime \prime}+a h_{1}^{\prime}\right) u_{2}, & \text { on } \operatorname{Supp}\left(\Omega_{1}\right), \\
a u_{2}^{\prime}-a h_{2}^{\prime} u_{2} & =a h_{2}^{\prime} u_{1}, & \text { on } \operatorname{Supp}\left(\Omega_{2}\right) .
\end{aligned}
$$

Under Assumption 1 and Equation (4), Equations (5) 2 and (3) $)_{3}$ are equivalent.

The problem we are solving is then equivalent, by Equation (3) 3 , to

$$
h_{1} v u^{\prime \prime}+a u^{\prime}=0, \quad \text { on }(0,1), \quad u(0)=0, u(1)=1,
$$


whose analytical solution, provided that $\operatorname{Supp}\left(\Omega_{1}\right)$ is connected, is given by

$$
u(x)=\frac{\int_{0}^{x}\left(e^{-\int_{0}^{y} \frac{a}{v h_{1}(z)} d z}\right) d y}{\int_{\operatorname{Supp}\left(\Omega_{1}\right)}\left(e^{-\int_{0}^{y} \frac{a}{v h_{1}(z)} d z}\right) d y}, \text { if } x \in \operatorname{Supp}\left(\Omega_{1}\right), u(x)=1, \text { otherwise. }
$$

We now study the approximation quality of this method as $\frac{v}{a} \rightarrow 0$ for a decreasing twice continuously differentiable membership function $h_{1}$ of the form

$h_{1}(x):=1$, if $0 \leq x \leq c_{1}, \quad h_{1}(x):=h(x)$, if $c_{1}<x<c_{2}, \quad h_{1}(x):=0$, if $c_{2} \leq x \leq 1$,

where $0<h(x) \leq 1$, so that $\operatorname{Supp}\left(\Omega_{1}\right)$ in Equation (12) is $\operatorname{Supp}\left(\Omega_{1}\right)=\left[0, c_{2}\right)$. We define $\delta:=c_{2}-c_{1}$ to be the width of the coupling region.

Theorem 2. For $h_{1}$ as in Equation (13), the relative error $\operatorname{err}_{A p p}\left(\frac{v}{a}\right):=\frac{\left\|u-u^{*}\right\|_{L^{2}(0,1)}}{\left\|u^{*}\right\|_{L^{2}(0,1)}}$ satisfies when $\frac{v}{a} \rightarrow 0$ the estimates:

\begin{tabular}{|c|c|c|c|c|}
\hline & $c_{1}=$ cst., & $c_{1}=\kappa\left(\frac{v}{a}\right)^{1-\varepsilon}$, & $c_{1}=\kappa \frac{v}{a} \ln \left(\frac{a}{v}\right)$, & $c_{1}=\kappa \frac{v}{a}$, \\
& $\delta=c s t$. & $\delta=\kappa^{\prime}\left(\frac{v}{a}\right)^{1-\varepsilon}$ & $\delta=\kappa^{\prime} \frac{v}{a}$ & $\delta=\kappa^{\prime} \frac{v}{a}$ \\
\hline $\operatorname{err}_{A p p}\left(\frac{v}{a}\right)$ & $O\left(e^{-\frac{a c_{1}}{v}}\right)$ & $O\left(e^{-\kappa\left(\frac{a}{v}\right)^{\varepsilon}}\right)$ & $O\left(\ln \left(\frac{a}{v}\right)^{0.5}\left(\frac{v}{a}\right)^{\kappa+0.5}\right)$ & $O\left(\left(\frac{v}{a}\right)^{0.5}\right)$ \\
\hline
\end{tabular}

Here, $\kappa>0, \kappa^{\prime} \geq 0$ are constants, and $0<\varepsilon \leq 1$.

Proof. The proof of this result is divided into 3 steps. Step 1 finds two functions $\tilde{u}_{1}^{*}$ and $\tilde{u}_{2}^{*}$ that satisfy $\tilde{u}_{1}^{*} \leq u \leq \tilde{u}_{2}^{*}$. With such functions, we always have the bound

$$
\frac{\left\|u-u^{*}\right\|_{L^{2}(0,1)}}{\left\|u^{*}\right\|_{L^{2}(0,1)}} \leq \max _{i=1,2} e_{i}, \quad e_{i}:=\frac{\left\|\tilde{u}_{i}^{*}-u^{*}\right\|_{L^{2}(0,1)}}{\left\|u^{*}\right\|_{L^{2}(0,1)}}
$$

Step 2 estimates $\max _{i=1,2} e_{i}^{2}$ and step 3 handles the 4 cases in (14).

Step 1: With $h_{1}$ as in Equation (13), we can express the function $u$ as

$$
u(x)= \begin{cases}\frac{1-e^{-\frac{a x}{v}}}{1-e^{-\frac{a c_{1}}{v}}\left(1-\frac{a}{v} \int_{c_{1}}^{c_{2}} e^{-\frac{a}{v} \int_{c_{1}}^{y} h^{-1}(z) d z} d y\right)}, & \text { if } 0 \leq x \leq c_{1}, \\ \frac{1-e^{-\frac{a c_{1}}{v}}\left(1-\frac{a}{v} \int_{c_{1}}^{x} e^{-\frac{a}{v} \int_{c_{1}}^{y} h^{-1}(z) d z} d y\right)}{1-e^{-\frac{a c_{1}}{v}}\left(1-\frac{a}{v} \int_{c_{1}}^{c_{2}} e^{-\frac{a}{v} \int_{c_{1}}^{y} h^{-1}(z) d z} d y\right)}, & \text { if } c_{1}<x<c_{2}, \\ 1, & \text { if } c_{2} \leq x \leq 1 .\end{cases}
$$

Using the fact that $0<h(z) \leq 1$, we have the estimate

$$
1-e^{-\frac{a c_{1}}{v}}<1-e^{-\frac{a c_{1}}{v}}\left(1-\frac{a}{v} \int_{c_{1}}^{x} e^{-\frac{a}{v} \int_{c_{1}}^{y} h^{-1}(z) d z} d y\right) \leq 1-e^{-\frac{a x}{v}}, \quad c_{1}<x<c_{2} .
$$

Using this estimate, we define $\tilde{u}_{i}^{*}, i=1,2$ as 


$$
\left.\begin{array}{l}
\text { if } 0 \leq x \leq c_{1}, \frac{1-e^{-\frac{a x}{v}}}{1-e^{-\frac{a c_{2}}{v}}} \\
\text { if } c_{1}<x<c_{2}, \frac{1-e^{-\frac{a c_{1}}{v}}}{1-e^{-\frac{a c_{2}}{v}}} \\
\text { if } c_{2} \leq x \leq 1,1
\end{array}\right\}=: \tilde{u}_{1}^{*}(x) \leq u(x) \leq \tilde{u}_{2}^{*}(x):= \begin{cases}\frac{1-e^{-\frac{a x}{v}}}{1-e^{-\frac{a c_{1}}{v}}} & \text { if } 0 \leq x \leq c_{1}, \\
\frac{1-e^{-\frac{a x}{v}}}{1-e^{-\frac{a c_{1}}{v}}} & \text { if } c_{1}<x<c_{2}, \\
1 & \text { if } c_{2} \leq x \leq 1\end{cases}
$$

Step 2: We now compute the relative $L^{2}$-errors for $\tilde{u}_{i}^{*}, i=1,2$. Using Equation (15), we have

$$
e_{1}^{2}=I_{1}(1,2)+I_{2}+I_{3} \quad \text { and } \quad e_{2}^{2}=I_{1}(2,1)+I_{3},
$$

where the different terms are integrals of the form $\int\left(\frac{\tilde{u}_{i}^{*}}{u}-1\right)^{2} d x$,

$$
\begin{gathered}
I_{1}(i, j):=\int_{0}^{c_{i}}\left(\frac{1-e^{-\frac{a}{v}}}{1-e^{-\frac{a c_{j}}{v}}}-1\right)^{2} d x=c_{i}\left(\frac{1-e^{-\frac{a}{v}}}{1-e^{-\frac{a c_{j}}{v}}}-1\right)^{2}=O\left(c_{i}\left(\frac{v}{a}\right) e^{-\frac{2 a c_{j}\left(\frac{v}{a}\right)}{v}}\right), \\
I_{2}:=\int_{c_{1}}^{c_{2}}\left[\frac{\left(1-e^{-\frac{a c_{1}}{v}}\right)\left(1-e^{-\frac{a}{v}}\right)}{\left(1-e^{-\frac{a c_{2}}{v}}\right)\left(1-e^{-\frac{a x}{v}}\right)}-1\right]^{2} d x \leq \delta \max _{i=1,2}\left(\left[\frac{\left(1-e^{-\frac{a c_{1}}{v}}\right)\left(1-e^{-\frac{a}{v}}\right)}{\left(1-e^{-\frac{a c_{2}}{v}}\right)\left(1-e^{-\frac{a c_{i}}{v}}\right)}-1\right]^{2}\right) \\
=O\left(\delta\left(\frac{v}{a}\right) e^{-\frac{2 a c_{1}\left(\frac{v}{a}\right)}{v}}\right), \\
I_{3}:=\int_{c_{2}}^{1}\left(\frac{1-e^{-\frac{a}{v}}}{1-e^{-\frac{a x}{v}}}-1\right)^{2} d x=\int_{c_{2}}^{1}\left[\sum_{k=1}^{\infty} e^{-\frac{k a x}{v}}\left(1-e^{-\frac{a}{v}}\right)-e^{-\frac{a}{v}}\right]^{2} d x=O\left(\frac{v}{a} e^{-\frac{2 a c_{2}\left(\frac{v}{a}\right)}{v}}\right) .
\end{gathered}
$$

As $e^{-\frac{a c_{i}}{v}}<1$ and $e^{-\frac{a x}{v}}<1$, we can use geometric series to obtain estimates of the different integrals. Taking only the leading term gives the result for $I_{1}(i, j)$ and $I_{3}$. For $I_{3}$, the leading term under the integration is $e^{-\frac{a x}{v}}$, because $x \leq 1$. For $I_{2}$ we also used the monotonicity of the exponential to obtain the bound and then, use once again a geometric series to conclude. In the order notation, we have specified the possible dependence of $c_{i}$ and $\delta$ on the parameter $\frac{v}{a}$.

Step 3: We now need to distinguish the different cases in order to complete the proof. Using Equations $(16,17,18)$, we can compute the results shown in Table 1. Finally, we use relation (15) to obtain (14).

This theorem shows that the approximation quality of the method is similar to the best known coupling methods for this kind of problem, namely the one based on the factorization of the operator, see [10].

Numerical experiment: We now show a numerical experiment, where we solve (10) with the membership function $h_{1}$ as in Equation (13), with

$$
h(x)=\delta^{-3}\left(2 x^{3}-3\left(c_{1}+c_{2}\right) x^{2}+6 c_{1} c_{2} x-c_{2}^{2}\left(3 c_{1}-c_{2}\right)\right),
$$

and $h_{2}:=1-h_{1}$. With this decomposition, we solve the advection-diffusion problem if $x \leq c_{1}$, the purely advective model if $x \geq c_{2}$, and the mixed model in-between. The coupling is done with a spline. We introduce a set of equidistant points $x_{i}=$ 


\begin{tabular}{|c|c|c|c|c|}
\hline & $\begin{array}{c}c_{1}=\text { cst., } \\
\delta=\text { cst. }\end{array}$ & $\begin{array}{c}c_{1}=\kappa\left(\frac{v}{a}\right)^{1-\varepsilon}, \\
\delta=\kappa^{\prime}\left(\frac{v}{a}\right)^{1-\varepsilon}\end{array}$ & $\begin{array}{c}c_{1}=\kappa \frac{v}{a} \ln \left(\frac{a}{v}\right), \\
\delta=\kappa^{\prime} \frac{v}{a}\end{array}$ & $\begin{array}{c}c_{1}=\kappa \frac{v}{a}, \\
\delta=\kappa^{\prime} \frac{v}{a}\end{array}$ \\
\hline$I_{1}(1,2)$ & $O\left(e^{-\frac{2 a c_{2}}{v}}\right)$ & $O\left(e^{-2\left(\kappa+\kappa^{\prime}\right)\left(\frac{a}{v}\right)^{\varepsilon}}\right)$ & $O\left(\ln \left(\frac{a}{v}\right)\left(\frac{v}{a}\right)^{2 \kappa+1}\right)$ & $O\left(\frac{v}{a}\right)$ \\
\hline$I_{1}(2,1)$ & $O\left(e^{-\frac{2 a c_{1}}{v}}\right)$ & $O\left(e^{-2 \kappa\left(\frac{a}{v}\right)^{\varepsilon}}\right)$ & $O\left(\ln \left(\frac{a}{v}\right)\left(\frac{v}{a}\right)^{2 \kappa+1}\right)$ & $O\left(\frac{v}{a}\right)$ \\
\hline$I_{2}$ & $O\left(e^{-\frac{2 a c_{1}}{v}}\right)$ & $O\left(e^{-2 \kappa\left(\frac{a}{v}\right)^{\varepsilon}}\right)$ & $O\left(\left(\frac{v}{a}\right)^{2 \kappa+1}\right)$ & $O\left(\frac{v}{a}\right)$ \\
\hline$I_{3}$ & $O\left(e^{-\frac{2 a c_{2}}{v}}\right)$ & $O\left(e^{-2\left(\kappa+\kappa^{\prime}\right)\left(\frac{a}{v}\right)^{\varepsilon}}\right)$ & $O\left(\left(\frac{v}{a}\right)^{2 \kappa+1}\right)$ & $O\left(\frac{v}{a}\right)$ \\
\hline$e_{1}^{2}$ & $O\left(e^{-\frac{2 a c_{1}}{v}}\right)$ & $O\left(e^{-2 \kappa\left(\frac{a}{v}\right)^{\varepsilon}}\right)$ & $O\left(\ln \left(\frac{a}{v}\right)\left(\frac{v}{a}\right)^{2 \kappa+1}\right)$ & $O\left(\frac{v}{a}\right)$ \\
\hline$e_{2}^{2}$ & $O\left(e^{-\frac{2 a c_{1}}{v}}\right)$ & $O\left(e^{-2 \kappa\left(\frac{a}{v}\right)^{\varepsilon}}\right)$ & $O\left(\ln \left(\frac{a}{v}\right)\left(\frac{v}{a}\right)^{2 \kappa+1}\right)$ & $O\left(\frac{v}{a}\right)$ \\
\hline
\end{tabular}

Table 1 Table of the order of the different integrals $I_{j}$.

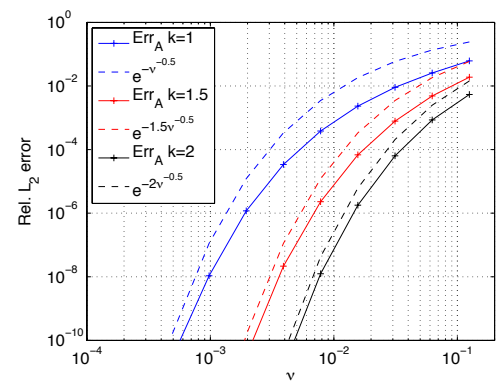

(a) Case 2: $c_{1}=k \frac{v}{a}^{1-\varepsilon}, \delta=\frac{v}{a}^{1-\varepsilon}$, with $a=$ $1, \varepsilon=0.5$ and $k=1,1.5,2$.

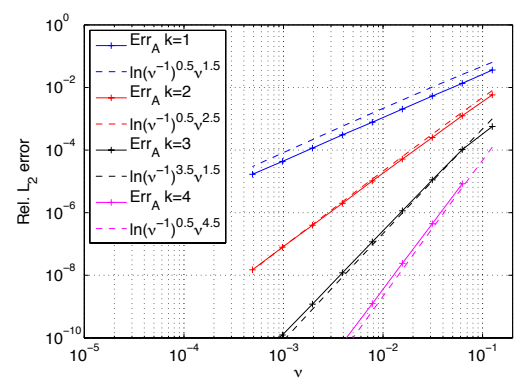

(b) Case 3: $c_{1}=k \frac{v}{a} \ln \left(\frac{a}{v}\right), \delta=\frac{v}{a}$, with $a=1$ and $k=1,2,3,4$.

Fig. 1 Results for the cases 2 and 3 of Theorem 2 where we refined the grid keeping $n v$ constant. We see that the curves follow the theoretical predictions.

$i \cdot \Delta x$ with $i=0, \ldots, n+1$ and $\Delta x=1 /(n+1)$. We discretize the problem (10) with an upwind 3-point finite difference scheme. This gives us a system of $2 n$ coupled equations. For each component $u_{j}, j=1,2$, we remove from the system all the irrelevant equations, those for which $h_{j}\left(x_{i}\right)=0$; this corresponds to the restriction to $\operatorname{Supp}\left(\Omega_{j}\right)$.

In order to illustrate the behavior of the method, we have chosen the cases 2 and 3 in Theorem 2. In both cases, the observed behavior is in very good agreement with the predictions, see Figure 1 where we computed the relative error $E r r_{A}$ between the numerical advection-diffusion solution and its approximation for different parameters. In the two cases shown, the coupling region is moving towards zero when $v$ is decreasing and we see that the approximation quality depends on how the coupling region is moved, accordingly to Theorem 2 . We kept $n v$ constant in order to capture the boundary layer that forms when $v \rightarrow 0$. 


\section{Conclusion}

We presented a new heterogeneous domain decomposition method based on Fuzzy Set Theory. We have shown a concise analysis for a simple, but relevant, model problem which showed that this type of coupling leads to a very efficient heterogeneous domain decomposition method. This method can be viewed as a formalisation of a coupling technique for very complex problems, see for example [5, 6] for the coupling between kinetic and hydrodynamic equations. In such a coupling, the partition between the different fuzzy domains can evolve with time and can even adapt automatically to the local conditions using some local criterion, see [6].

We think that such methods have a great potential in various coupling problems and in particular for problems in which the partition into different domains of validity of concurrent approximations is not a priori clear, because they permit to try different criteria by changing only the way the membership functions are defined.

We are currently interested in such a method for the coupling of the diffusion limit of the relativistic Boltzmann equation with a stationary free streaming limit of it. This would be an alternative to the current version of the IDSA, which still has some mathematical issues that need to be fixed, see [2,3] for more details.

\section{References}

1. Achdou, Y., Pironneau, O.: The $\chi$-method for the Navier-Stokes equations. IMA J. Numer. Anal. 13(4), 537-558 (1993)

2. Berninger, H., Frénod, E., Gander, M., Liebendörfer, M., Michaud, J.: Derivation of the IDSA for supernova neutrino transport by asymptotic expansions. Submitted to SIMA (2012)

3. Berninger, H., Frénod, E., Gander, M., Liebendörfer, M., Michaud, J., Vasset, N.: A mathematical description of the IDSA for supernova neutrino transport, its discretization and a comparison with a finite volume scheme for Boltzmann's equation. In: Esaim: Proceedings, vol. 38, pp. 163-182 (2012)

4. Brezzi, F., Canuto, C., Russo, A.: A self-adaptive formulation for the Euler/Navier-Stokes coupling. Comput. Methods Appl. Mech. Engrg. 73(3), 317-330 (1989)

5. Degond, P., Dimarco, G., Mieussens, L.: A moving interface method for dynamic kinetic fluid coupling. J. Comput. Phys. 227, 1176-1208 (2007)

6. Degond, P., Dimarco, G., Mieussens, L.: A multiscale kinetic-fluid solver with dynamic localization of kinetic effects. J. Comput. Phys. 229, 4907-4933 (2010)

7. Degond, P., Jin, S.: A smooth transition between kinetic and diffusion equations. SIAM J. Numer. Anal. 42(6), 2671-2687 (2005)

8. Degond, P., Jin, S., Mieussens, L.: A smooth transition model between kinetic and hydrodynamic equations. J. Comput. Phys. 209, 665-694 (2005)

9. Discacciati, M., P., G., Quarteroni, A.: Heterogeneous mathematical models in fluid dynamics and associated solution algorithms. Tech. Report MOX 04/2010 (2010)

10. Gander, M.J., Martin, V.: An asymptotic approach to compare coupling mechanisms for different partial differential equations. In: Domain Decomposition Methods in Science and Engineering XX, Lect. Notes Comput. Sci. Eng. Springer (2012). In print

11. Liebendörfer, M., Whitehouse, S., Fischer, T.: The isotropic diffusion source approximation for supernova neutrino transport. Astrophys. J. 698, 1174-1190 (2009)

12. Zadeh, L.: Fuzzy Sets. Information and Control 8(3), 338-353 (1965) 